MAGNETOHYDRODYNAMICS Vol. 54 (2018), No. 1-2, pp. 49-53

DOI: $10.22364 /$ mhd.54.1-2.9

\title{
INFLUENCE OF LONGITUDINAL MAGNETIC FIELD ON THE KELVIN-HELMHOLZ INSTABILITY FOR TWO LAYERS OF FERROFLUID AT RELATIVE HORIZONTAL MOTION
}

\author{
V.M.Korovin ${ }^{1}$, V.A.Kazhan ${ }^{2}$ \\ 1 Moscow State University, Research Institute of Mechanics, \\ 1 Michurinsky pr., 117192 Moscow, Russia \\ ${ }^{2}$ Russian State Agrarian University, \\ 49 Timiryazevskaya str., 127550 Moscow, Russia \\ e-Mail:verazhan@yandex.ru
}

The motion of two horizontal layers of nonlinearly magnetizable inviscid ferrofluids which have a plane interface is considered. A criterion of such motion stability is found.

Introduction. A criterion of the Kelvin-Helmholz instability for the case of relative motion of two layers of ferrofluids has been found in [1] using a linear magnetization law. In this paper, we investigate this instability without any restriction to the value of the applied magnetic field. A neutral stability curve is obtained for the case of nonlinear magnetization. We consider a ferrofluids, whose magnetization is described by the modified Langevin law [2]. The stability regions for the Langevin and linear magnetization cases are compared.

1. Problem formulation. We consider two superimposed layers of inviscid immiscible ferrofluids with different physical parameters. The layers move with constant velocities in the direction of the applied magnetic field $\mathbf{H}_{0}$. The indices $j=1,2$ indicate the bottom and top layers of the ferrofluids, respectively. At the initial time $t=0$, the interface is plane. Let $O x y z$ be a Cartesian coordinate system, where the $x$-axis is parallel to $\mathbf{H}_{0}$ and located at the plane interface, and the $z$-axis is directed vertically upwards (see Fig. 1). Let $\mathbf{a}_{x}, \mathbf{a}_{y}, \mathbf{a}_{z}$ stand for the corresponding unit coordinate vectors and $u_{0 j} \mathbf{a}_{x}$ for the layers' velocities. The equations $z=\zeta(x, y, t), t>0$ describes the shape of the interface in the presence of waves. The wave amplitudes are small if compared with their lengths. The fluids' flow is assumed to be potential.

We use the common notations: $\mathbf{B}_{j}(x, y, z, t)=\mu_{j} \mathbf{H}_{j}=\mu_{0}\left(\mathbf{M}_{j}+\mathbf{H}_{j}\right)$ is the magnetic induction, $\mathbf{H}_{j}(x, y, z, t)=\nabla \psi_{j}$ is the magnetic field, $\mathbf{M}_{j}(x, y, z, t)=\chi_{j} \mathbf{H}_{j}$ is the magnetization vector, $\mu_{j}\left(H_{j}\right)=\mu_{0}\left[1+\chi_{j}\left(H_{j}\right)\right]$ denotes permeability, $\mu_{0}=$ $4 \pi \cdot 10^{-7} \mathrm{H} / \mathrm{m}$ is the magnetic constant. Here we take into account the dependence of the magnetic susceptibility $\chi_{j}$ on $H_{j}=\sqrt{H_{j x}^{2}+H_{j y}^{2}+H_{j z}^{2}}$. Also we use the differential permeability [1] $\mu_{t j}\left(H_{j}\right)=\mathrm{d} B_{j} / \mathrm{d} H_{j}$ and the differential magnetic susceptibility $\chi_{t j}\left(H_{j}\right)=\mathrm{d} M_{j} / \mathrm{d} H_{j}$, where $\mu_{t j}\left(H_{j}\right)=\mu_{0}\left[1+\chi_{t j}\left(H_{j}\right)\right]$.

The problem is assumed to be linear. Let $\psi_{j}(x, y, z, t)=H_{0} x+f_{j}(x, y, z, t)$, $\mathbf{u}_{j}(x, y, z, t)=u_{0 j} \mathbf{a}_{x}+\nabla \varphi_{j}(x, y, z, t)$, where $f_{j}, \varphi_{j}$ are the small perturbation potentials of magnetic fields and velocities. To find $f_{j}$, one needs to solve the 
following magnetostatics problem:

$$
\begin{gathered}
\nabla^{2} f_{j}+\left[\sigma_{j}\left(H_{0}\right)-1\right] \frac{\partial^{2} f_{j}}{\partial x^{2}}=0, \quad \sigma_{j}\left(H_{0}\right)=\frac{1+\chi_{t j}\left(H_{0}\right)}{1+\chi_{j}\left(H_{0}\right)}, j=1,2 \\
z=0: f_{1}=f_{2}, \quad \mu_{1}^{0} \frac{\partial f_{1}}{\partial z}-\mu_{2}^{0} \frac{\partial f_{2}}{\partial z}=\mu_{0}\left(M_{01}-M_{02}\right) \frac{\partial \zeta}{\partial x} \\
z \rightarrow-\infty: \nabla f_{1} \rightarrow 0, \quad z \rightarrow+\infty: \quad \nabla f_{2} \rightarrow 0
\end{gathered}
$$

where

$$
\nabla=\frac{\partial}{\partial x} \mathbf{a}_{x}+\frac{\partial}{\partial y} \mathbf{a}_{y}+\frac{\partial}{\partial z} \mathbf{a}_{z}, \quad \mu_{j}^{0}=\mu_{j}\left(H_{0}\right), \quad M_{0 j}=M_{j}\left(H_{0}\right), j=1,2 .
$$

The equation for $f_{j}$ is a special case of the equation derived in [3]. From the plots of the experimental magnetization curves, it follows that for the sufficiently strong fields $\chi_{j}\left(H_{0}\right)>\chi_{t j}\left(H_{0}\right)$. Hence, $\sigma_{j}\left(H_{0}\right)<1$. In case of linear magnetization (weak fields case), $\sigma_{j}=1$.

The fluid motion is described by the Laplace equations. At $z=0$, two kinematic conditions and the condition of pressure continuity should be fulfilled. For the latter the Cauchy-Lagrange integrals for each fluid are used:

$$
\begin{aligned}
\nabla^{2} \varphi_{j} & =0, j=1,2 ; \quad z=0: \quad \frac{\partial \zeta}{\partial t}+u_{0 j} \frac{\partial \zeta}{\partial x}=\frac{\partial \varphi_{j}}{\partial z}, \quad j=1,2 \\
z=0: & \rho_{2}\left(\frac{\partial \varphi_{2}}{\partial t}+u_{02} \frac{\partial \varphi_{2}}{\partial x}\right)-\rho_{1}\left(\frac{\partial \varphi_{1}}{\partial t}+u_{01} \frac{\partial \varphi_{1}}{\partial x}\right)-g \zeta\left(\rho_{1}-\rho_{2}\right) \\
& +a\left(\frac{\partial^{2} \zeta}{\partial x^{2}}+\frac{\partial^{2} \zeta}{\partial y}\right)+\mu_{0}\left(M_{01} \frac{\partial f_{1}}{\partial x}-M_{02} \frac{\partial f_{2}}{\partial x}\right)=0 \\
z \rightarrow-\infty & : \nabla \varphi_{1} \rightarrow 0 ; \quad z \rightarrow+\infty: \nabla \varphi_{2} \rightarrow 0
\end{aligned}
$$

Here $\rho_{1}>\rho_{2}$ are the fluid densities, $g$ is the gravitational acceleration, $\alpha$ is the surface tension.

2. Stability investigation. Let us investigate the dependence of the solutions $\zeta(x, y, t), f_{j}(x, y, z, t), \varphi_{j}(x, y, z, t)$ of problems (1), (3) on $t$ by means of normal mode analysis [4]. Let us use the normal modes proportional to $\exp \left\{i\left[k_{x} x+\right.\right.$ $\left.\left.k_{y} y-\omega\left(k_{x}, k_{y}\right) t\right]\right\}$, where $i$ is an imaginary unit, $\mathbf{k}=k_{x} \mathbf{a}_{x}+k_{y} \mathbf{a}_{y}$ is the wave

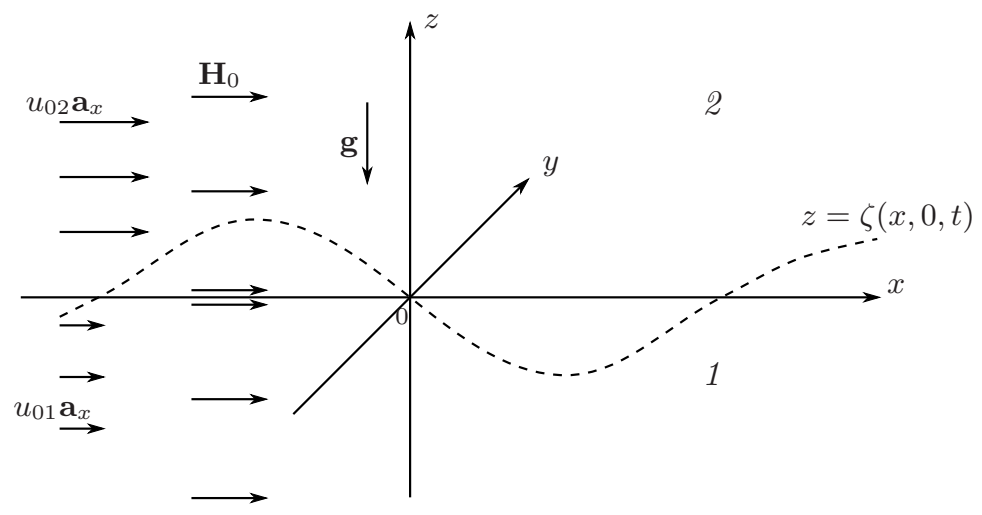

Fig. 1. Geometry of the problem. 
Influence of longitudinal magnetic field on the Kelvin-Helmholz instability for ...

vector, $k_{x}^{2}+k_{y}^{2} \neq 0$. The corresponding dispersion equation is tedious, so, to simplify the investigation of its roots $\omega_{1,2}\left(k_{x}, k_{y}\right)$, we use the polar coordinates $k, \theta$ : $k_{x}=k \cos \theta, k_{y}=k \sin \theta, 0 \leq \theta \leq 2 \pi$. Hence,

$$
\begin{aligned}
\omega_{1,2}\left(k_{x}, k_{y}\right) & =\frac{\rho_{1} u_{01}+\rho_{2} u_{02}}{\rho_{1}+\rho_{2}} k \cos \theta \pm \sqrt{\frac{k}{\rho_{1}+\rho_{2}} W(k, \tau)} \\
W(k, \tau) & =\alpha k^{2}+g\left(\rho_{1}-\rho_{2}\right)+\tau s(\tau) k, \tau=\cos ^{2} \theta, 0 \leq \tau \leq 1, u_{0}=u_{02}-u_{01}, \\
s(\tau) & =\frac{\mu_{0}^{2}\left(M_{01}-M_{02}\right)^{2}}{\mu_{1}^{0} \sqrt{1+\left(\sigma_{1}-1\right) \tau}+\mu_{2}^{0} \sqrt{1+\left(\sigma_{2}-1\right) \tau}}-\frac{\rho_{1} \rho_{2} u_{0}^{2}}{\rho_{1}+\rho_{2}} .
\end{aligned}
$$

It is shown that for all $k>0,0 \leq \tau \leq 1$, and when

$$
u_{0}<q\left(H_{0}\right) \equiv\left\{\frac{\rho_{1}+\rho_{2}}{\rho_{1} \rho_{2}}\left[2 \sqrt{\alpha g\left(\rho_{1}-\rho_{2}\right)}+\frac{\mu_{0}\left(M_{1}\left(H_{0}\right)-M_{2}\left(H_{0}\right)\right)^{2}}{2+\chi_{1}\left(H_{0}\right)+\chi_{2}\left(H_{0}\right)}\right]\right\}^{\frac{1}{2}},
$$

one has $W(k, \tau)>0$. Thus, the roots of the dispersion equation are real and the motion of the layers with a plane interface is stable. For the case of linear magnetization from Eq. (5) it follows

$$
u_{0}<\left\{\frac{\rho_{1}+\rho_{2}}{\rho_{1} \rho_{2}}\left[2 \sqrt{\alpha g\left(\rho_{1}-\rho_{2}\right)}+\frac{\mu_{0}\left(\chi_{\iota 1}-\chi_{\iota 2}\right)^{2}}{2+\chi_{\iota 1}+\chi_{\iota 2}} H_{0}^{2}\right]\right\}^{\frac{1}{2}},
$$

where $\chi_{\iota j}=\lim _{H_{0} \rightarrow 0} \chi_{j}\left(H_{0}\right), j=1,2$ are the initial magnetic susceptibilities.

The curve $u_{0}=q\left(H_{0}\right)$ is the neutral stability curve which divides the first quadrant of the $\left(H_{0}, u_{0}\right)$ plane into the stability and instability regions. The stability region is located under the curve and the instability region is located above the curve.

As an example, we consider a ferrofluid whose magnetization is described by the modified Langevin law proposed in [2]:

$$
M_{j}\left(H_{j}\right)=M_{s j} L\left(3 \chi_{\iota j} H_{j} / M_{s j}\right)
$$

where $M_{s j}$ is the saturation magnetization and $L(a)=\operatorname{coth} a-1 / a$. For weak fields when $0<H_{j} \ll M_{s j} /\left(3 \chi_{\iota j}\right)$, one has $M_{j}\left(H_{j}\right)=\chi_{\iota j} H_{j}\left[1-0.6\left(\chi_{\iota j} H_{j} / M_{s j}\right)^{2}\right]$.

Let us consider a case, where at the bottom, there is a ferrofluid which was used in the experimental study [5]. The parameters of this ferrofluid are $M_{s 1}=16.9 \mathrm{kA} / \mathrm{m}, \chi_{\iota 1}=0.69, \rho_{1}=1324 \mathrm{~kg} / \mathrm{m}^{3}, \alpha_{1}=0.059 \mathrm{~N} / \mathrm{m}$. It is assumed that the top layer consists of a ferrofluid with the physical parameters $M_{s 2}=31.8 \mathrm{kA} / \mathrm{m}$, $\rho_{2}=1250 \mathrm{~kg} / \mathrm{m}^{3}, \alpha_{2}=0.028 \mathrm{~N} / \mathrm{m}$ (see Table 2.4 in [1]). We adopt $\chi_{\iota 2}=0.9$ as a characteristic value.

In Fig. 2, the Langevin magnetization curves for the top and bottom ferrofluids are shown by solid curves 1, 2. The dashed lines stay for the linear magnetization case.

In Fig. 3, the neutral stability curves corresponding to the magnetization curves in Fig. 2 are represented by a solid and a dashed line, respectively. We used the characteristic value $\alpha=0.059 \mathrm{~N} / \mathrm{m}$. For the case of weak fields, the curves in Fig. 3 show a negligible difference of the $u_{0}$ range, where the motion of the layers with a plane interface is stable. It is seen from Fig. 3 that the linear magnetization law produces a smaller $u_{0}$ range compared to the Langevin magnetization law. For the strong fields, the linear magnetization law is not satisfied. In this case, the stability region is described by Eq. (5). 
V.M. Korovin, V.A.Kazhan

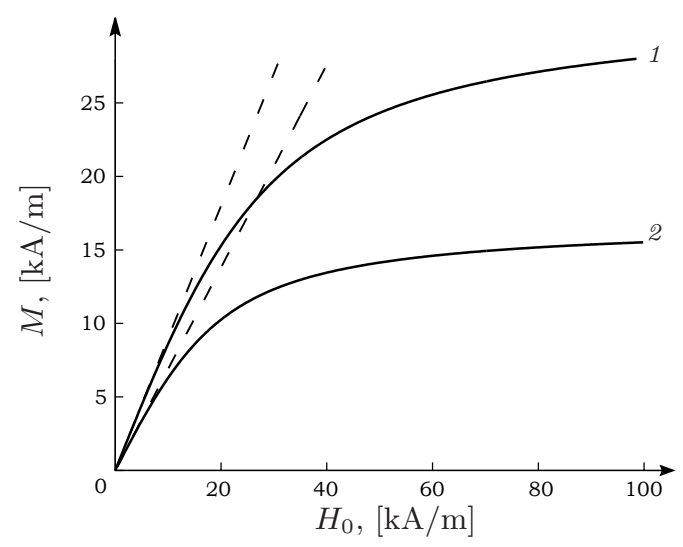

Fig. 2. Magnetization law.

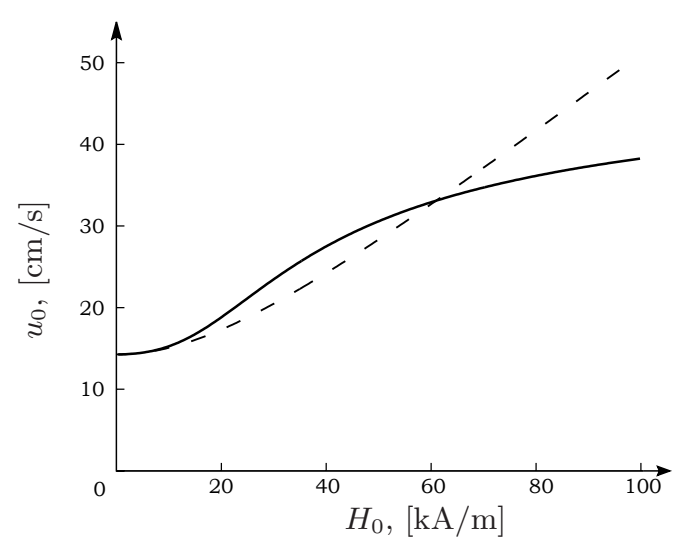

Fig. 3. Neutral stability curves.

3. Conclusion. An equation for the neutral stability curve is derived with the following parameters: the field strength and the relative velocity of the layers. The case of ferrofluids whose magnetization is described by the modified Langevin law [2] is considered in detail. It is shown that for the case of strong fields one has to use the derived equation for the neutral stability curve to find a relative velocity range belonging to the stability region.

Acknowledgements. The study was supported by the RFBR (project no. 1701-00037).

\section{References}

[1] R.E. Rosensweig. Ferrohydrodynamics (Cambridge University Press, New York, 1985).

[2] B. Abou, G. Néron de Surgy, J.E. Wesfreid. Dispersion relation in a ferrofluid layer of any thickness and viscosity in a normal magnetic field; asymptotic regimes. J. Phys. II France, vol. 7 (1997), no. 8, pp. 1159-1171.

[3] V.M. Korovin. On the influence of a horizontal magnetic field on the Rosensweig instability of a nonlinear magnetizable ferrofluid. Technical Physics, vol. 59 (2014), no. 11, pp. 1577-1584. DOI: 10.1134/S1063784214110152. 
Influence of longitudinal magnetic field on the Kelvin-Helmholz instability for ...

[4] P.G. Drazin. Introduction to Hydrodynamic Stability. (Cambridge University Press, Cambridge, 2002).

[5] S. Dorbolo, E. FAlcon. Wave turbulence on the surface of a ferrofluid in a horizontal magnetic field. Phys. Rev. E., vol. E83 (2011), 046303.

Received 27.12.2017 